\title{
Fingerprinting differential active site constraints of ATPases
}

\author{
Stephan M. Hacker, $\neq^{\text {a }}$ Norman Hardt,$\neq^{\text {a }}$ Alexander Buntru, ${ }^{\text {b }}$ Dana Pagliarini, ${ }^{\text {b }}$ \\ Martin Möckel, ${ }^{\mathbf{b}}$ Thomas U. Mayer, $^{\mathbf{b}}$ Martin Scheffner, ${ }^{\mathbf{b}}$ Christof R. Hauck ${ }^{\mathbf{b}}$ \\ and Andreas Marx ${ }^{\star a}$
}

The free energy provided by adenosine triphosphate (ATP) hydrolysis is central to many cellular processes and, therefore, the number of enzymes utilizing ATP as a substrate is almost innumerable. Modified analogues of ATP are a valuable means to understand the biological function of ATPases. Although these enzymes have evolved towards binding to ATP, large differences in active site architectures were found. In order to systematically access the specific active site constraints of different ATPases suitable tools are required. Here, we present the synthesis of six new ATP-based ATPase probes modified at three different positions of the nucleobase and the ribose, respectively. Subsequently, we studied the ATPases focal adhesion kinase FAK, the ubiquitin-activating protein UBA1 and the kinesin Eg 5 as examples for ATPases that process ATP by different mechanisms. We find that for each of these enzymes at least one position in ATP can be modified without loss of acceptance by the enzyme. However, the positions at which modifications are tolerated significantly differ between the studied enzymes allowing fingerprints to be drawn for reactivity. The introduced ATP analogues may form the basis for the design of tailored probes with increased affinity and specificity for a specific ATPase of interest.

\section{Introduction}

In the post-genomic era, one of the great challenges is to dissect the function and regulation of cellular molecules. ${ }^{1}$ In this context, adenosine and its nucleotides play a central role since the free energy provided by ATP hydrolysis is central to many cellular processes and, therefore, the number of enzymes utilizing ATP as a substrate is almost innumerable. ${ }^{2}$ Although they all bind ATP, different enzyme families have evolved very distinct structural elements to optimize their binding. ${ }^{3}$ Therefore, knowledge about the specific ATP binding site architecture and its functional constraints of ATP-processing enzymes (ATPases) is pivotal to elucidate their mechanisms of action.

Analogues of ATP have proven themselves as invaluable probes to elucidate the mechanism of reactions catalyzed by ATP-dependent enzymes in depth. Mainly, modifications

"Department of Chemistry, Konstanz Research School Chemical Biology, University of Konstanz, Universitätsstr. 10, 78457 Konstanz, Germany. E-mail: Andreas.Marx@ uni-konstanz.de; Fax: +49753188 5140; Tel: +497531885139

${ }^{\circ}$ Department of Biology, Konstanz Research School Chemical Biology, University of Konstanz, Universitätsstr. 10, 78457 Konstanz, Germany

† S. M. H. and N. H. contributed equally. attached via an ester or carbamate bond at the $\mathrm{O}^{\prime}{ }^{\prime}$ - and $\mathrm{O}^{\prime}{ }^{\prime}$ position of the ribose have been used to study ATPases. One pioneering molecule in the field is $N$-methyl-anthraniloyl-ATP (mantATP). ${ }^{4}$ This fluorophore-conjugated ATP analogue has been widely used to study the kinetics of binding and release of ATP to various enzymes using the change of fluorescence intensity as a readout. ${ }^{5}$ Recently, alternative fluorophoreconjugated ATP analogues have been used to overcome the drawbacks of the short wavelength absorption and emission of mantATP. ${ }^{6}$ Furthermore, these analogues have also been helpful to monitor the distance between the ATP binding site and a fluorophore labeled DNA substrate in the case of a bacterial transcription regulatory protein using the Förster resonance energy transfer (FRET) mechanism. ${ }^{7}$

Besides dyes, further modifications of ATP include photocrosslinking reagents, such as aryl azides or benzophenones introduced by ester or carbamate attachment to the $\mathrm{O}^{\prime}$ - and $O 3^{\prime}$-position. These analogues can be used to affinity label the active site of ATPases and in this way to gain information on active site architecture. ${ }^{8}$ Spin-labels were also introduced to ATP for investigation of ATPases in EPR studies. ${ }^{9}$ Furthermore, affinity purification of certain ATPases using biotin-labeled ATP analogues has been accomplished. ${ }^{10}$

All the abovementioned ATP analogues comprising ester bonds have the drawback that labels attached to the $O 2^{\prime}$ - and $O 3^{\prime}$-position of ATP isomerize quickly. ${ }^{11}$ Using a carbamate linkage this isomerization can be slowed down, but obtaining 
pu re isomers is still difficult. ${ }^{12}$ Oiwa et al. could nicely show that - at least in the case of a Cy3 labeled ATP analogue - the two isomers can have very different spectroscopic features complicating data analysis. ${ }^{13}$

Attachment sites other than the $O 2^{\prime}$ - or $O 3^{\prime}$-position have been exploited in a few cases. ${ }^{14}$ However, most of these ATP probes are conditioned by the synthetic availability of their modifications rather than adjustment to the biological system of interest. A possibility to study the best attachment site to ATP and in this way to optimize the probes towards a specific ATPase is still missing.

The goal in this study is to systematically explore the active site constraints of different ATPases using a set of ATP analogues that bear modifications at different positions within the nucleotide. In this way, fingerprints of the site-specific demands of different ATP binding sites will be obtained. Therefore, we synthesized ATP analogues with modifications at three different positions of the nucleobase as well as the $O 2^{\prime}-$-, $O 3^{\prime}$ - and $C^{\prime} 4^{\prime}$-position of the ribose, respectively (Fig. 1A).

We set out to synthesize analogues bearing a trifluoroacetamide group at the chosen positions. This modification was used since it can be easily converted to the free amine by deprotection. In this way, the six molecules presented here will also allow straightforward further modification by usage of amine-reactive chemistries.

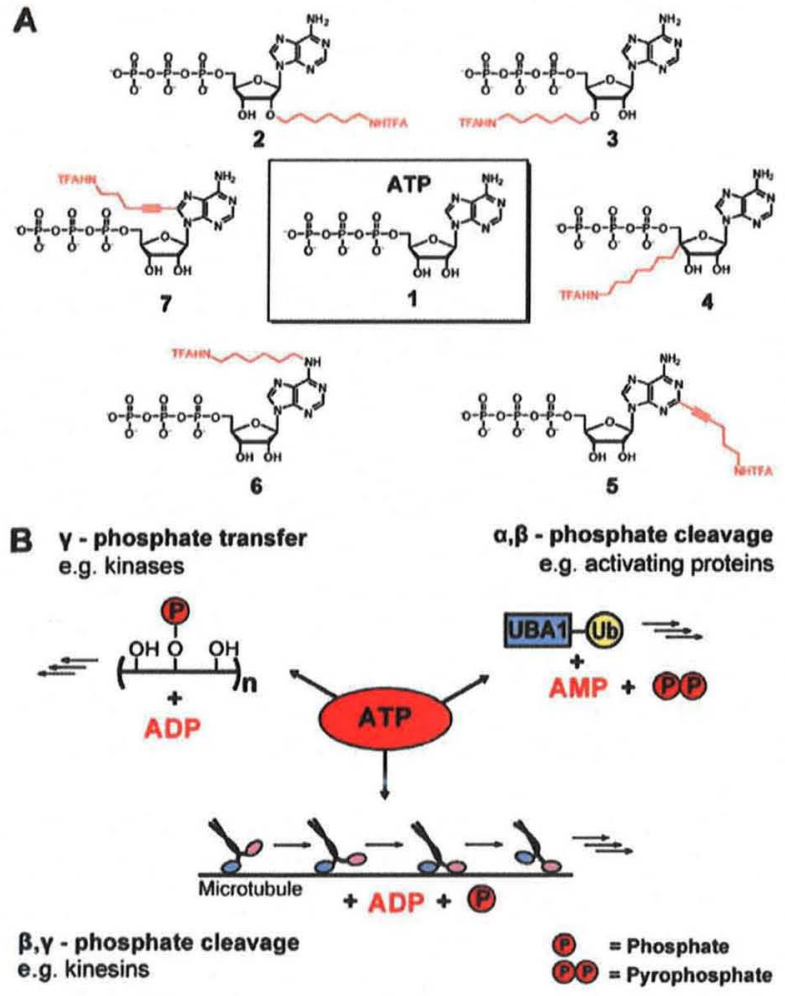

Fig. 1 (A) Natural ATP (1) and six novel ATP analogues (2-7) used in this study. All analogues bear a modification fused to a trifluoroacetamide group at three different positions of the nucleobase as well as at the $O 2^{\prime}-, \mathrm{O}^{\prime}$ and $\mathrm{CL}^{\prime}$-position of the ribose, respectively. (B) Turnover of ATP by the three classes of ATPases including one representative of phosphor transferases, $\alpha, \beta$ - and $\beta, \gamma$-phosphate cleaving enzymes investigated in this study.
Subsequently, we evaluated the ATP analogues on their ability to be used by different classes of ATPases. We chose to investigate three enzymes that belong to mechanistically different types of ATPases and, therefore, process ATP by different modes of action (Fig. 1B). The focal adhesion kinase (FAK) belongs to the family of protein tyrosine kinases and was chosen as an example for an enzyme that cleaves the $\beta$ - $\gamma$-phosphoanhydride bond and transfers the $\gamma$-phosphate of ATP to a substrate. ${ }^{15}$ The kinesin Eg5 is representative of kinesin motor proteins and processes the $\beta$ - $\gamma$-phosphoanhydride bond. It uses the energy released by this cleavage to undergo structural changes and in this way to migrate along microtubules. ${ }^{16}$ The ubiquitin-activating enzyme UBA1 is the initial protein starting the ubiquitylation process and cleaves the $\alpha-\beta$-phosphoanhydride bond and uses the energy to form a thioester that is used to activate ubiquitin towards conjugation. ${ }^{17}$ We find that for each of these enzymes at least one position in ATP can be modified without loss of acceptance by the enzyme. However, the positions at which modifications are tolerated significantly differ.

\section{Results and discussion}

\section{Synthesis of ATP analogues}

In order to explore suitable sites for modifications within the ATP skeleton we chose the $\mathrm{O}^{\prime}{ }^{\prime}-\mathrm{O}^{\prime}$ - and $\mathrm{C4}^{\prime}$-positions, respectively, since they were expected to interfere less with conformations of the glycosidic bond and the triphosphate in comparison to modifications of positions $C 1^{\prime}$ and $C 5^{\prime}$.

Positions $C 2, N 6$ and $C 8$ were chosen for nucleobase modifications since the positions of the nitrogen atoms within the heterocycle are retained.

The synthesis of ATP analogues modified at the $O 2^{\prime}$ - and $O 3^{\prime}$ started with adenosine (Scheme 1). Up to now, $\mathrm{O2}^{\prime}$ - and $\mathrm{O3}^{\prime}$ modified ATP probes have mainly been used as a mixture of both isomers as a consequence of isomerisation of the modification attached by an ester or carbamate bond. ${ }^{11}$ Thus, we synthesized the stable ether modifications of these sites.

The synthesis of $O 2^{\prime}$-(6-(trifluoro-acetamido)-hexyl)-adenosine triphosphate (2) started with the treatment of adenosine with $\mathrm{NaH}$ and the conversion with 6-azido-1-bromohexane to give $36 \%$ of $O 2^{\prime}$-(6-azidohexyl)-adenosine (2a). ${ }^{18}$ Compound 2a was converted to the corresponding amine via a Staudinger reduction $^{19}$ and subsequent TFA protection was performed using ethyl trifluoroacetate to give $O 2^{\prime}$-(6-(trifluoroacetamido)hexyl)-adenosine (2b) in $93 \%$ yield. The corresponding triphosphate 2 was obtained in $57 \%$ yield following a one-pot procedure. $^{20}$

For the synthesis of $\mathrm{O3}^{\prime}$-(6-(trifluoroacetamido)-hexyl)-adenosine triphosphate (3), adenosine was $O 2^{\prime}$-, $O 5^{\prime}$ - and N6-trityl protected using tritylchloride and pyridine to yield adenosine $3 \mathbf{a}$ in $45 \% .{ }^{21}$ Compound 3 a was treated with $\mathrm{NaH}$ and reacted with 6 azido-1-bromohexane to give $3 \mathbf{b}$ in a yield of $50 \% .^{22}$ The protection groups were removed with $80 \%$ acetic acid, the azide was reduced with triphenylphosphine in a Staudinger reduction ${ }^{19}$ and the resulting free amine was subsequently TFA-protected with ethyl trifluoroacetate to give $\mathrm{O}^{\prime}$-(6-(trifluoroacetamido)hexyl)-adenosine (3d) in a yield of $33 \%$ over three steps. 

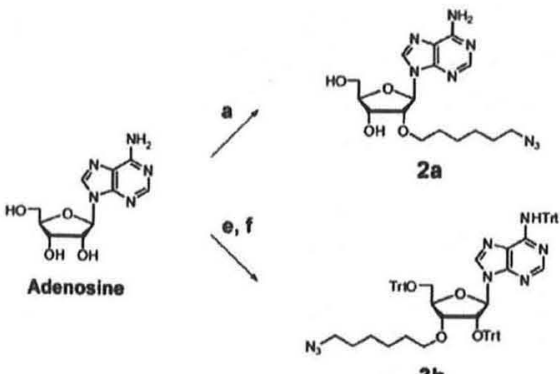

3b

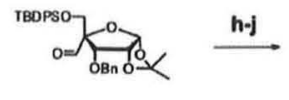

4 a

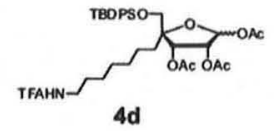

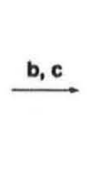
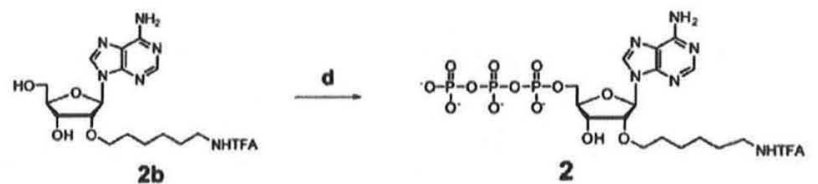

$\stackrel{\text { g, b, c }}{\longrightarrow}$
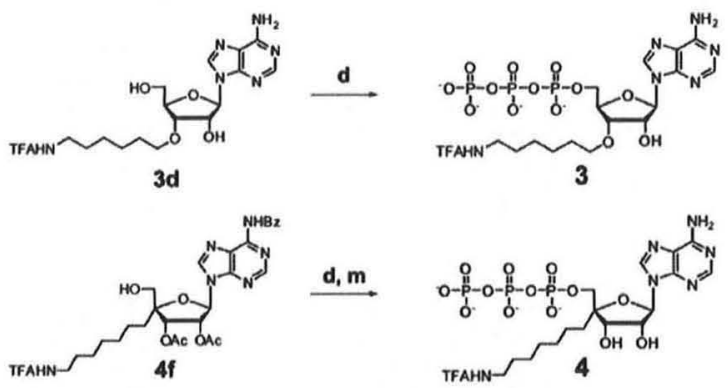

d, $\mathbf{m}$

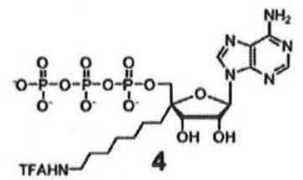

Scheme 1 Synthesis of three novel ribose modified ATP analogues (2-4). (a) 6-Azido-1-bromohexane, NaH, DMF, r.t., 24 h. (b) PPh, THF, H ${ }_{2} \mathrm{O}$, r.t., 24 h. (c) Ethyl trifluoroacetate, $\mathrm{Et}_{3} \mathrm{~N}, \mathrm{MeOH}$, r.t., 24 h. (d) (1) $\mathrm{POCl}_{3}$, proton sponge, $\mathrm{TMP}, \mathrm{O}^{\circ} \mathrm{C}, 1$ h, (2) $\left(\mathrm{Bu}_{3} \mathrm{NH}^{+}\right)_{2} \mathrm{H}_{2} \mathrm{P}_{2} \mathrm{O}_{7}{ }^{2-}, \mathrm{Bu}_{3} \mathrm{~N}$, r.t., $30 \mathrm{~min}$, (3) $0.1 \mathrm{M} \mathrm{TEAB}$, pH= 7.5, r.t., 30 min. (e) Trt-Cl, pyridine, $100^{\circ} \mathrm{C}, 4$ h. (f) 6-Azido-1-bromohexane, $\mathrm{NaH}, \mathrm{ACN}$, r.t., 48 h. (g) $80 \% \mathrm{AcOH}, 110^{\circ} \mathrm{C}, 90$ min. (h) Triphenyl-(6-(trifluoroacetamido)hexyl)-phosphonium iodide, $t$-BuOK, THF, r.t., 2 h. (i) $\mathrm{H}_{2} / \mathrm{Pd}$, THF, r.t., 2 h. (j) $\mathrm{ACOH} / \mathrm{AC} \mathrm{C}_{2} \mathrm{O}$, r.t., 24 h. (k) (1) $\mathrm{N}^{6}$-Bz-adenine, BSA, ACN, reflux, 2 h, $0^{\circ} \mathrm{C},(2) \mathrm{TMSO}-\mathrm{Tf}$, reflux, 24 h. (I) TBAF, AcOH, THF, r.t., 24 h. (m) (1) $33 \% \mathrm{NH}_{3}$ aq., r.t., 3.5 h. (2) Ethyl trifluoroacetate, $\mathrm{MeOH}_{\text {, }} \mathrm{NEt}_{3}$, r.t., 3 h.

Triphosphate 3 was obtained from $3 d$ in $49 \%$ yield following a one-pot procedure..$^{20}$

The modification at the $C 4^{\prime}$-position was introduced by the de novo synthesis of $C 4^{\prime}$-(7-(trifluoroacetamido)-heptyl)-modified adenosine triphosphate (4) starting with compound 4a that was synthesized in a five step synthesis as reported. ${ }^{23}$ The aldehyde at the C4-position was converted to a modification containing the trifluoroacetamide moiety via Wittig reaction using triphenyl-(6-(trifluoroacetamido)-hexyl)-phosphonium iodide that was synthesized in a four-step procedure starting from 6-amino-1-hexanol, ${ }^{24}$ followed by hydrogenation using $10 \% \mathrm{Pd} / \mathrm{C}$ under $\mathrm{H}_{2}$ atmosphere. Subsequent protection group manipulations using $\mathrm{AcOH} / \mathrm{Ac}_{2} \mathrm{O}$ and catalytic amounts of $\mathrm{H}_{2} \mathrm{SO}_{4}$ yielded the acetyl protected ribose $4 \mathrm{~d}$ in $72 \%$ over three steps. Glycosylation was performed via the Vorbrüggen approach using N6-benzoyl protected adenine. ${ }^{25}$ The protected adenosine bearing a modification at the $C 4^{\prime}$-position was obtained in $50 \%$ yield. The main side product could be identified as N7-linked adenosine. By deprotection of the 5 -hydroxyl group using $1 \mathrm{M}$ TBAF solution, compound af could be obtained in $63 \%$. Compound af was used for triphosphate synthesis following a one-pot procedure in $14 \%{ }^{20}$ The resulting triphosphate was deprotected using $33 \% \mathrm{NH}_{3}(\mathrm{aq})-\mathrm{H}_{2} \mathrm{O}(1: 2)$ to give $C 4^{\prime}$-(7-aminoheptyl)-modified adenosine triphosphate. Next, the amino group of the adenosine triphosphate was TFA protected using ethyl trifluoroacetate to give $C 4^{\prime}$-(7-(trifluoroacetamido)-heptyl) adenosine triphosphate (4) in a yield of $30 \%$ over the last two steps.

Next, we turned to the synthesis of ATP analogues modified at the nucleobase (Scheme $2 \mathrm{~A}$ ). To attach a modification to the $C 2$-position of the nucleobase, we synthesized 2-iodo-adenosine in a five-step synthesis according to Engels and colleagues starting from guanosine. ${ }^{26} \mathrm{~A}$ Sonogashira coupling reaction was performed with 5-trifluoroacetamido-pentyne to give C2-(5-trifluoroacetamido-pent-1-yn-1-yl)-adenosine (5a) in 93\% yield.
Triphosphate 5 was obtained following a standard procedure in $34 \%$ yield. ${ }^{20}$

N6-(6-(trifluoroacetamido)-hexyl) adenosine triphosphate (6) was synthesized starting with 6-chloro-9-( $\beta$-D-ribofuranosyl)purine (6a) that was synthesized over 3 steps in $65 \%$ starting from inosine. ${ }^{27}$ Treatment with 1,6-diaminohexane under alkaline condition ${ }^{28}$ followed by amino group protection with ethyl trifluoroacetate gave N6-(6-(trifluoroacetamido)-hexyl) adenosine (6c) in $48 \%$ yield over two steps. Triphosphate 6 was obtained using nucleoside $\mathbf{6 c}$ in $34 \%$ yield using a standard procedure. $^{20}$

C8-(5-trifluoroacetamido-pent-1-yn-1-yl) adenosine triphosphate (7) was synthesized starting from 8 -bromo-adenosine. ${ }^{29}$ Sonogashira coupling with 5-trifluoroacetamido-pentyne gave C8-(5-trifluoroacetamido-pent-1-yn-1-yl) adenosine (7a) in 83\% yield. Triphosphate 7 was obtained in $31 \%$ yield following a standard procedure. ${ }^{20}$

In principle, the presented ATP analogues are amenable to be converted to tailored ATPase probes by cleavage of the TFAamide to yield an aliphatic amine and subsequent coupling to a label of interest. To provide first proof along these lines we exemplarily investigated these conversions using the N6modified triphosphate 6 and the standard fluorescent dye Sulfo-Cy5 that was suitably modified in order to covalently connect both molecules yielding the labeled triphosphate $\mathbf{6 d}$ (Scheme 2B). The trifluoroacetamide of triphosphate 6 was deprotected using $0.1 \mathrm{M} \mathrm{NaOH}$ in water at room temperature yielding the free amine and leaving the triphosphate chain intact. This molecule can subsequently be reacted with the commercially available Sulfo-Cy5 $\mathrm{N}$-hydroxy succinimide (NHS) ester in situ. For this purpose, the reaction was adjusted to $\mathrm{pH}$ 8.7 using carbonate buffer and treated with the Sulfo-Cy5 NHS ester dissolved in DMF at room temperature. In this way, Sulfo-Cy5 labeled triphosphate $6 \mathrm{~d}$ was obtained in $45 \%$ yield over two steps. 
A
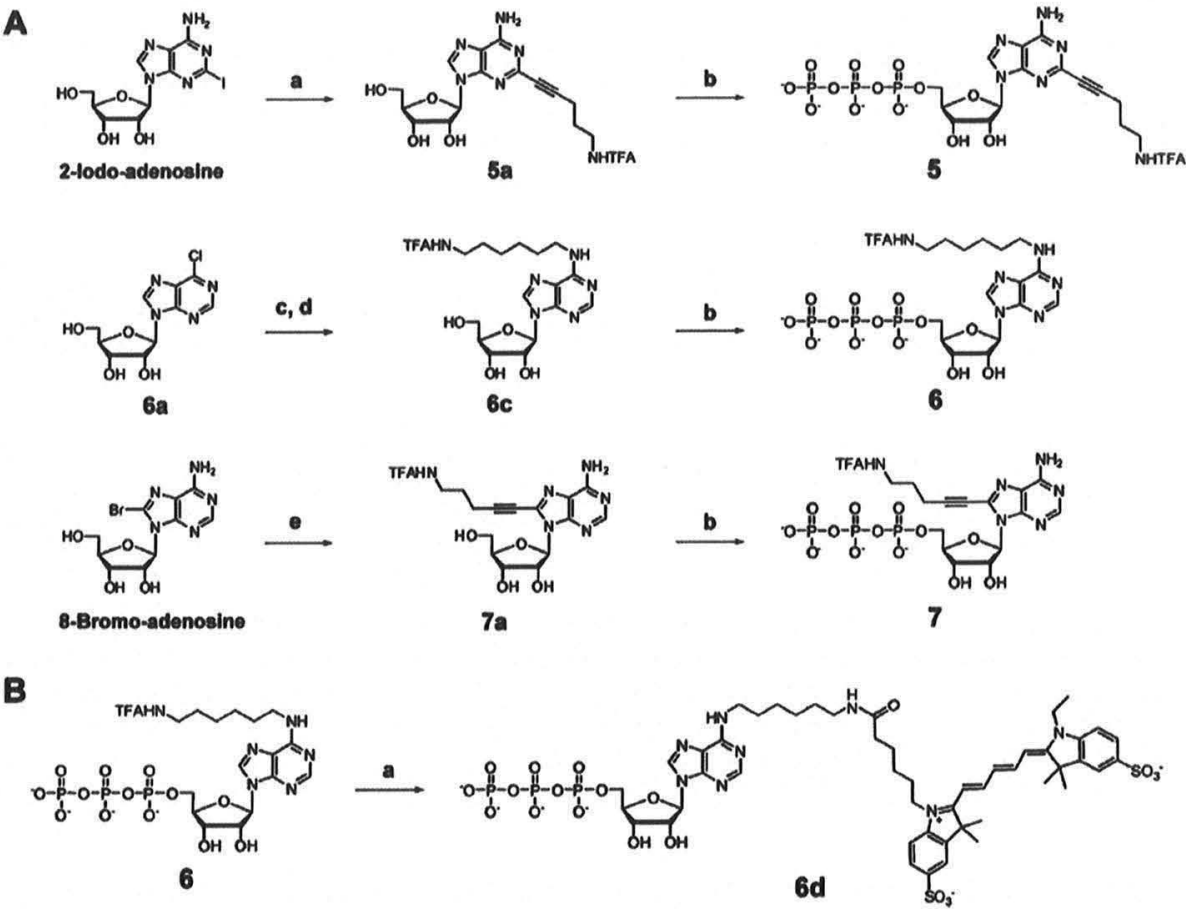

Scheme 2 (A) Synthesis of three novel nucleobase modified ATP analogues (5-7). (a) 5-Trifluoroacetamido-pentyne, Pd(PPh $)_{4}, \mathrm{Cul}, \mathrm{NEt} 3, \mathrm{DMF}, \mathrm{r.t.}, 24 \mathrm{~h}$. (b) (1) POCl 3 , proton sponge, TMP, $0^{\circ} \mathrm{C}, 1 \mathrm{~h}$, (2) $\left(\mathrm{Bu}_{3} \mathrm{NH}^{+}\right)_{2} \mathrm{H}_{2} \mathrm{P}_{2} \mathrm{O}_{7}{ }^{2-}, \mathrm{Bu}_{3} \mathrm{~N}$, r.t., $30 \mathrm{~min}$, (3) $0.1 \mathrm{M} \mathrm{TEAB}, \mathrm{pH}=7.5$, r.t., 30 min. (c) 1,6-Diaminohexane, Et ${ }_{3} \mathrm{~N}, \mathrm{EtOH}$, reflux, $3 \mathrm{~h}$. (d) Ethyl trifluoroacetate, $\mathrm{Et}_{3} \mathrm{~N}, \mathrm{MeOH}$, r.t., 24 h. (e) 5-Trifluoroacetamido-pentyne, $\mathrm{PdCl}_{2}\left(\mathrm{PPh}_{3}\right)_{2}, \mathrm{Cul}_{2} \mathrm{Et}_{3} \mathrm{~N}, \mathrm{DMF}$, r.t., 24 h. (B) Synthesis of the dye labelled analogue $6 \mathrm{~d}$. (a) (i) $0.1 \mathrm{M} \mathrm{NaOH}, 4$ h, RT, (ii) Sulfo-Cy5 NHS ester, $0.1 \mathrm{M} \mathrm{NaHCO}_{3}, \mathrm{DMF}, \mathrm{pH} 8.7$, r.t., 12 h.

\section{Biochemical evaluation of ATP analogues}

Having the six novel trifluoroacetamide-modified triphosphates available, we turned to the investigation of their acceptance by different ATPases and started with an example of a protein kinase. The approximately 500 protein kinases encoded in the human genome comprise one of the largest protein families and together control virtually every cellular process. ${ }^{30}$ Protein kinases catalyze the transfer of the $\gamma$-phosphate group of ATP onto their substrate proteins and this reversible modification often serves as a switch to alter the functionality of the substrate protein. In multicellular animals the subset of protein tyrosine kinases (PTKs), which transfer the $\gamma$-phosphate to tyrosine residues of their substrates, have a key role in signal transduction, regulating cell proliferation and other cellular behaviour. Accordingly, deregulated PTK activity is often causally involved in diseases such as cancer making PTKs a prime target for therapeutics. ${ }^{31}$ As a prototype PTK, we chose to investigate FAK, which is activated upon cell adhesion and mediates signal transduction downstream of integrins. ${ }^{32}$

To evaluate the ability of FAK to utilize the modified ATP analogues we employed an in vitro kinase assay based on the recombinant FAK kinase domain and a peptide substrate (Fig. 2A). ${ }^{33}$ To this end, we immobilized a surrogate FAK substrate peptide (poly-(Glu:Tyr); 4:1) in 96-well plates. Modified ATP analogues were added to the substrate either with or without kinase and incubated at $37^{\circ} \mathrm{C}$. Phosphorylation of poly-(Glu-Tyr) was detected by a phosphotyrosine-specific antibody in combination with a secondary antibody coupled to horseradish-peroxidase (HRP). HRP activity was then measured by oxidation of tetramethylbenzidine (TMB) as a read-out for FAK-mediated tyrosine phosphorylation.

The results of these in vitro kinase assays show significant differences between the modified ATP analogues and their use by FAK (Fig. 2B). Specifically, modifications at $\mathrm{O2}^{\prime}$-position (2), $O 3^{\prime}$-position (3) and C2-position (5) of ATP are tolerated by this PTK. In contrast, the $C 4^{\prime}$-modified (4), N6-modified (6), and $C 8$ modified (7) analogues are not used as a co-substrate by FAK.

To further investigate the ability of FAK to utilize ATP analogues, we conducted a time course analysis (Fig. 2C). A linear increase of the target phosphorylation can be seen in this experiment for all investigated ATP analogues allowing quantification of the substrate properties of the different ATP analogues. While $\mathrm{O}^{\prime}$-modified ATP (2) shows about $70 \%$ of the phosphorylation activity of natural ATP, the $03^{\prime}$ modified analogue (3) shows only about $40 \%$. Most importantly, using the ATP analogue bearing a 5-(pent-1-yn-1-yl)-trifluoroacetamide at the $C 2$-position (5) almost $90 \%$ of the kinase activity observed with natural ATP can be reached.

Next, we investigated the acceptance of the synthesized ATP analogues by the kinesin motor protein Eg5 that cleaves the $\beta-\gamma$ phosphoanhydride bond. This protein is a key player in the establishment of the bipolar spindle apparatus and therefore essential for correct cell division..$^{34}$ In its catalytic cycle a highly coordinated series of ATP binding, ATP hydrolysis, ADP and phosphate release events catalyzed by the motor domain leads 

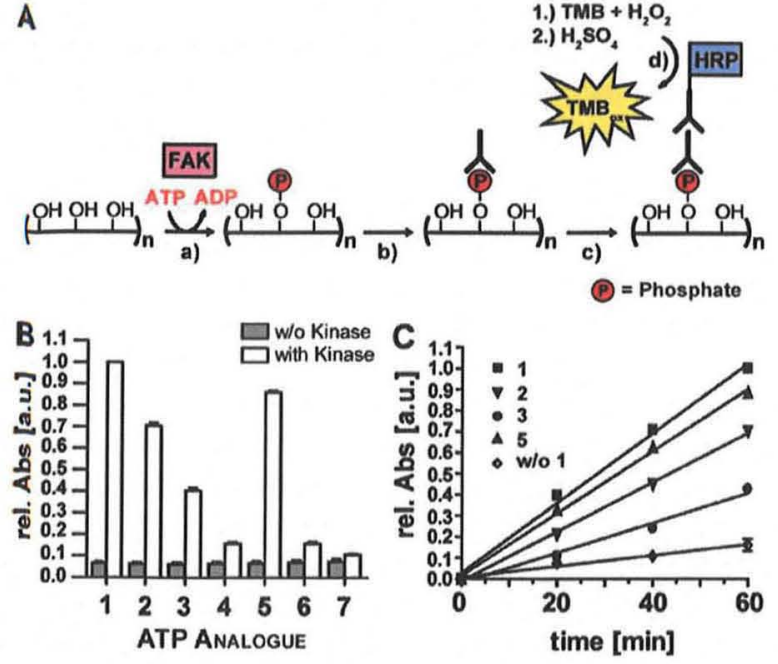

Fig. 2 (A) Schematic depiction of the FAK in vitro kinase assay using modified ATPanalogues. (a) Incubation of immobilized, tyrosine-rich peptide substrate, FAK, and the ATP of interest at $37^{\circ} \mathrm{C}$. (b) Immobilized substrate is incubated with antiphosphotyrosine antibody. (c) The primary anti-phosphotyrosine-antibody is detected by a HRP-conjugated secondary antibody. (d) HRP oxidizes TMB to its oxidated form $\mathrm{TMB}_{\mathrm{ox}}$. The reaction was quenched after 3 min with $2 \mathrm{M} \mathrm{H}_{2} \mathrm{SO}_{4}$ and the absorption of $\mathrm{TMB}_{\text {ox }}$ measured at $450 \mathrm{~nm}$. (B) Absorption of $\mathrm{TMB}_{\mathrm{ox}}$ in the reaction solutions of the indicated ATP analogue after 1 hour of incubation. The absorptions were normalized to unmodified ATP (1). Negative controls were performed without addition of the kinase. Values represent mean + SEM of three independent experiments. (C) Absorption of $\mathrm{TMB}_{\mathrm{ox}}$ in the reaction solutions of the indicated ATP analogue after incubation for indicated time intervals. The absorptions were normalized to incubation with unmodified ATP (1) for 1 hour. Values represent mean \pm SEM of three independent experiments done in triplicates. Negative controls were performed without ATP. The $\mathrm{OZ}^{\prime}$-modified (2), $\mathrm{O}^{\prime}$-modified (3) and C2-modified (5) analogues can clearly serve as a co-substrate for FAK.

to migration along microtubules towards the plus end (Fig. 3A). ${ }^{35}$ Due to its tetrameric structure, Eg5 is supposed to be able to crosslink and slide apart antiparallel microtubules within the mitotic spindle. Inhibition of Eg5 by the small molecule compound Monastrol in vivo has widely helped to understand the process of spindle bipolarization. ${ }^{36}$

We examined the tolerance of the monomeric motor-domain of Eg5 for the different ATP analogues using a reported malachite green assay. ${ }^{37}$ The Eg5-mediated release of $\gamma$-phosphate can be quantified by detecting the change in absorption at $650 \mathrm{~nm}$ due to complex formation of malachite green with phosphate. We measured the absorption after 0 minutes and 6 minutes of incubation and calculated the acceptance by Eg 5 for each analogue as the difference between both values (Fig. 3B). Negative controls were performed without Eg5. Additionally, we evaluated the time-dependence of the reaction (Fig. 3C). The reaction was stopped by addition of perchloric acid at indicated time points. The rise of absorbance in all reactions is linear over time showing that the measurements were performed in the linear portion of the kinetic curve.

The results revealed that modification at the ribose moiety of ATP at all positions tested is well accepted by Eg5. Up to 70\% of the activity observed with natural ATP can be retained using the $O 2^{\prime}$-modified analogue 2 . Interestingly, the $C 4^{\prime}$-modified
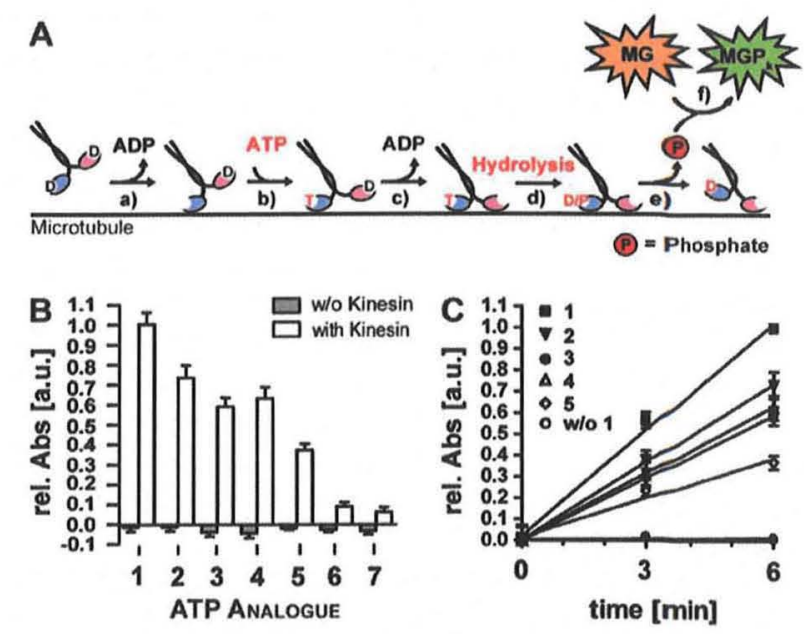

Fig. 3 (A) Schematic depiction of the ATPase assay for the activity assay of ATP analogues towards Eg5. (a)-(e) Using a highly coordinated series of ADP release, ATP binding, ATP hydrolysis and phosphate release events $\mathrm{Eg} 5$ can migrate along microtubules. ( $f$ ) After quenching with perchloric acid the release of phosphate can be detected by addition of malachite green and measurement of the increasing absorption of the malachite green phosphate complex at $650 \mathrm{~nm}$. (B) All ATP analogues were treated with $\mathrm{Eg} 5$ and microtubules and the reaction was quenched after $0 \mathrm{~min}$ and $6 \mathrm{~min}$ at ambient. Relative absorption was calculated as the difference between the two values and normalized to natural ATP (1). Negative controls were performed without kinesin. Values represent mean + SEM of three independent experiments. (C) All ATP analogues were treated with Eg5 and microtubules and the reaction was quenched after $0 \mathrm{~min}, 3 \mathrm{~min}$ and $6 \mathrm{~min}$ at ambient. Relative absorption was calculated by subtracting the value at $0 \mathrm{~min}$ and normalized to the value after incubation for $6 \mathrm{~min}$ with natural ATP (1). Negative controls were performed without ATP. Values represent mean \pm SEM of three independent experiments. The $O 2^{\prime}$-modified (2), $\mathrm{O}^{\prime}$-modified (3), $\mathrm{CH}^{\prime}$-modified (4) and C2-modified (5) ATP analogues can clearly act as substrate for Eg5.

derivative 4 is also comparably active in this assay, whereas this analogue is not utilized by FAK. In addition, some activity can be seen using the $C 2$-modified analogue 5 . However, compared to FAK, Eg5 was less efficient in using analogue 5 as substrate. The N6-modified (6) and C8-modified (7) analogues were almost completely inactive in this system.

To show that the high degree of acceptance observed for ATP analogue 2 correlates with the ability of Eg5 to migrate along microtubules we performed a microtubule-gliding assay (ESI, Fig. S1 $\dagger$ ). In this assay, full length Eg5 is absorbed on a glass slide and the Eg5-mediated movement of fluorescently labelled microtubules is determined. As expected from our malachite green studies, the observed microtubule velocity is almost identical in the presence of ATP or $\mathrm{O2}^{\prime}$-modified ATP analogue 2 (ESI, Fig. S1†). The absolute value is in good agreement with the reported value for unmodified ATP. ${ }^{36}$ In contrast, no motility of the microtubules can be observed if no ATP is supplied. To ensure that the motility of the microtubules is indeed caused by Eg5 we additionally performed an inhibition experiment using Monastrol. The motility is inhibited by Monastrol to a large degree in the presence of ATP as well as in the presence of modified analogue 2 unambiguously demonstrating that the motility is exclusively caused by Eg5 activity. 
Finally, we investigated the tolerance of the ATP analogues by the ubiquitin (Ub)-activating enzyme UBA1 that cleaves the $\alpha$ $\beta$-phosphoanhydride bond of ATP to activate Ub by first Ubad enylate formation followed by Ub-thioester formation with UBA1. UBA1 is the initial enzyme in the ubiquitin conjugation system which modifies numerous target proteins by the covalent attachment of ubiquitin ("ubiquitylation"). As ubiquitylation has been involved in the regulation of many, if not all, fundamental cellular processes, it is not surprising that deregulation of components of the ubiquitin-conjugation system contributes to the development of various human disorders including cancer and neurodegenerative disorders. ${ }^{38}$ Accordingly, UBA1 has been considered as target in the treatment of various types of cancer. ${ }^{39}$

In the initial step of the ubiquitin conjugation cascade, UBA1 activates ubiquitin by conjugation to the $\alpha$-phosphate group of ATP releasing pyrophosphate and then transfers ubiquitin from the adenylate to its catalytic cysteine residue by forming a thioester bond (Fig. 4A). This initiates transfer of ubiquitin to a cysteine of a ubiquitin conjugating enzyme (E2). Finally, ubiquitin can be transferred to a target protein with the help of a ubiquitin ligase (E3). ${ }^{40}$
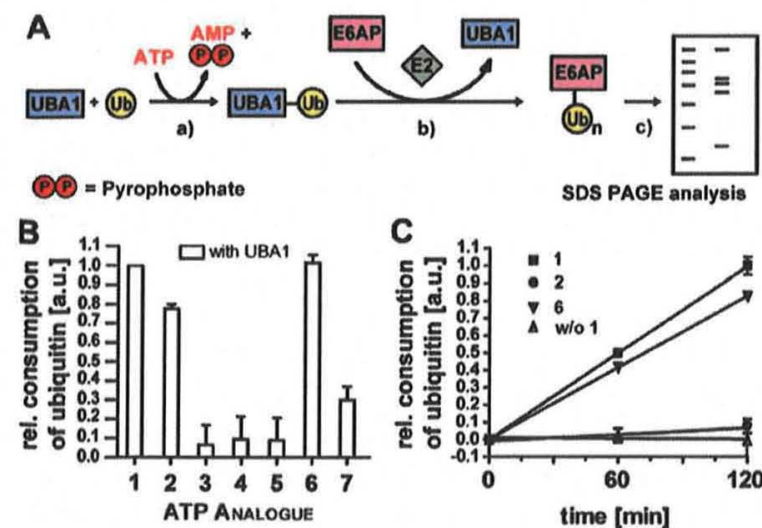

SDS PAGE analysis

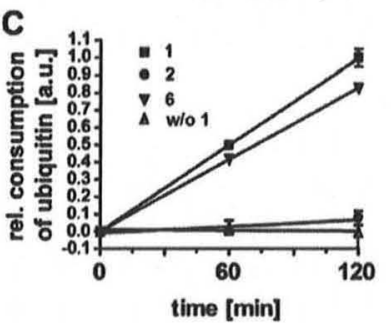

Fig. 4 (A) Schematic depiction of the assay to study the acceptance of the ATP analogues by UBA1. (a) UBA1 activates ubiquitin in an ATP-dependent manner. (b) This activation initiates the transfer of ubiquitin via the E2 to E6AP. Polyubiquitin chains are formed on E6AP accompanied by the consumption of nonmodified E6AP and ubiquitin. (c) All three processes can be monitored by SDSPAGE followed by Coomassie staining. (B) All ATP analogues were incubated in the presence of $150 \mathrm{nM} \mathrm{UBA} 1, \mathrm{UbCH} 5 \mathrm{~b}$ (E2), E6AP and ubiquitin for $1 \mathrm{~h}$ at $37^{\circ} \mathrm{C}$. The intensity of the remaining ubiquitin band was quantified. Consumption of ubiquitin was calculated relative to incubation without ATP and this value was normalized to natural ATP. Values represent mean + SEM of two independent experiments. (C) All ATP analogues were incubated in the presence of a rate limiting amount (10 nM) UBA1, UbcH5b (E2), E6AP and ubiquitin for indicated time intervals at $37{ }^{\circ} \mathrm{C}$. The intensity of the remaining ubiquitin band was measured. Consumption was calculated relative to incubation for 0 hours and this value was normalized to the incubation with natural ATP for 2 hours. Values represent mean \pm SEM of two independent experiments. As judged by the strong disappearance of free ubiquitin the N6-modified analogue 6 is best accepted in this system. At a high E1 concentration activity can also be seen with the $\mathrm{O}^{\prime}$ '-modified ATP $\mathbf{2}$ and to a lesser extent with the C8-modified ATP 7. This activity can hardly be seen even for $\mathrm{O2}^{\prime}$-modified ATP 2 at a rate-limiting E1 concentration. This shows that this analogue is significantly less well tolerated than analogue 6 .
To study the acceptance of the modified ATP analogues by UBA1 we performed an E6AP autoubiquitylation assay. ${ }^{41}$ E6AP is an E3 ligase that has been causally associated with the development of three different human disorders - cervical cancer, Angelman syndrome, and autism spectrum disorders - and, thus, understanding and monitoring its activity is of considerable biomedical interest. ${ }^{42}$ In the auto-ubiquitylation assay, ubiquitin is activated as described above. In the last step, E6AP serves as both E3 ligase and target protein resulting in its autoubiquitylation with numerous ubiquitin moieties. This can be monitored in a coomassie stained SDS-PAGE by a decrease in the levels of non-modified E6AP and free ubiquitin and concomitant appearance of polyubiquitylated forms of E6AP that do not enter the gel. After incubation in the absence of ATP, E6AP and ubiquitin are still present, whereas both bands vanish almost completely after incubation with ATP accompanied by a rise of polyubiquitylated E6AP (ESI, Fig. S2†). To quantify the amount of ubiquitylation that takes place in the reactions we decided to quantify the remaining band of free ubiquitin (Fig. 4B). The N6-modifed ATP 6 is the most active as detected by

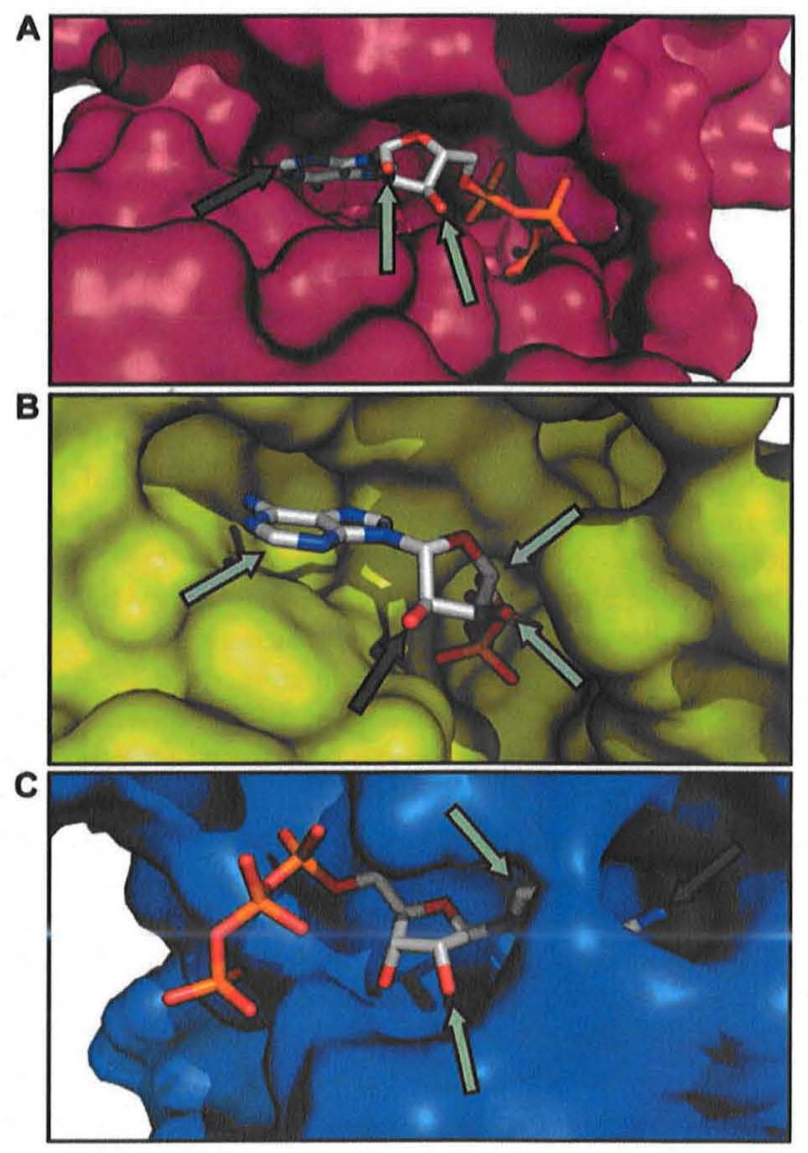

Fig. 5 (A) Close-up view of FAK (purple, Connolly surface, PDB: 2IJM) with ATP (sticks) bound to the active site. (B) View into the active site of Eg5 (yellow, Connolly surface, PDB: 1116) with ADP (sticks) bound. (C) Active site of UBA5 (blue, Connolly surface, PDB: $3 \mathrm{H} 8 \mathrm{~V}$ ) with ATP (sticks) attached. Dark green arrow: position, at which modification is tolerated best; light green arrow positions, at which modification is also accepted. 
almost the same level of depletion of the band representing free ubiquitin as for natural ATP. Of note, this ATP analogue has proven to be completely inactive with the two other ATPases tested. Additionally, some reduction of the ubiquitin band is detected with the $O 2^{\prime}$-modified analogue 2 and the $C 8$-modified analogue 7. Nevertheless, as judged by the lower amount of consumed ubiquitin, less ubiquitin was transferred in these cases indicating that these modifications are less well tolerated by UBA1. All other modifications at the $O 3^{\prime}$-position (3), $C 4^{\prime}$ position (4), and $C 2$-position (5) turned out to be inactive with UBA1.

To quantify the utilization of the ATP analogues by UBA1 we performed a time course analysis (Fig. 4C). As the ubiquitylation process is a multi-enzyme cascade we decided to work at a ratelimiting concentration of $10 \mathrm{nM}$ UBA1 in this case. In this way a direct quantification of the degree of utilization of the ATP analogues should be possible. One can see that even using these stringent conditions the N6-modified analogue $\mathbf{6}$ is almost as well tolerated as natural ATP. Nevertheless, the second best analogue, $O 2^{\prime}$-modified ATP 2, does not show high turnover of ubiquitin in this case.

\section{Conclusions}

In conclusion, we synthesized six novel adenosine triphosphate analogues. We chose to modify ATP at three different positions of the nucleobase $\left(\mathrm{C} 2, \mathrm{~N} 6\right.$ and $\mathrm{C} 8$ ) as well as at the $\mathrm{O}^{\prime}{ }^{\prime}-, \mathrm{O}^{\prime}{ }^{\prime}$ - and $C 4^{\prime}$-position of the ribose. All molecules could be obtained in pure form without regioisomerisation. In each molecule the modifications are attached via stable carbon-oxygen-ether, carbon-nitrogen or carbon-carbon bonds that prohibit isomerisation or cleavage of the connection during the studies.

All of the modified ATP analogues were employed in three different enzymatic assays to investigate their substrate properties. To analyse distinct classes of ATPases we investigated the protein kinase FAK transferring the $\gamma$-phosphate to a peptide substrate, the kinesin Eg5 cleaving the $\beta$ - $\gamma$-phosphoanhydride bond and the ubiquitin-activating enzyme UBA1 cleaving the $\alpha$ $\beta$-phosphoanhydride bond, as they all belong to mechanistically distinct classes of ATPases. We were able to find modification sites that are very well tolerated for each of the tested enzymatic systems. Additionally, each modified ATP analogue synthesized turned out to be active with at least one of the enzymes.

For FAK and Eg5 we found that modification at the $\mathrm{O}^{\prime}{ }^{\prime}$ - and $O 3^{\prime}$-position is accepted. This result is in good agreement with the widespread use of $O 2^{\prime}$ - or $O 3^{\prime}$-ester or carbamate modified ATP analogues to investigate kinases and kinesins..$^{\mathbf{5}, \mathbf{6}, \mathbf{8}, \mathbf{9}}$ Nevertheless, using the depicted strategy to design new enzymatic probes could result in more stable and homogeneous molecules. Furthermore, at least in the case of FAK and UBA1 a large difference in acceptance of the $O 2^{\prime}$ - and $O 3^{\prime}$-modification was observed showing that one regioisomer is more active than the other.

In addition, we found that FAK tolerates $C 2$-modified ATP analogues best. Due to the high conservation of active sites within the kinase family ${ }^{43}$ we predict that these results will be valid not only for FAK, but also for other enzymes within this family. Interestingly, only in the case of Eg5 we found that $C 4^{\prime}$-modified ATP analogues are well accepted. This class of probes has not been described before and could open the way to probes that can specifically address kinesins in the presence of other ATPases.

In contrast, UBA1 has a very different substrate spectrum. UBA1 can accept $O 2^{\prime}$-modified ATP analogues as the other enzymes, but also shows activity with $C 8$-modified analogues and has a strong preference for modification at the position $N 6$. The latter two modification sites are inactive in both other systems studied. These results indicate that the binding pocket of this class of enzymes is very different from the other ATPases. Furthermore, modification at the N6-position was proposed to result in ATP analogues that cannot be used as substrate for major classes of ATPases including kinases and kinesins. ${ }^{44}$ In this way such analogues could be a very valuable starting point for the design of specific probes for UBA1. Indeed, the acceptance of N6-modified ATP analogues by UBA1 is in good agreement with the usage of 6-modified adenosine analogues as ATP competitive inhibitors for UBA1 and homologous enzymes. ${ }^{45}$

Interestingly, the biochemical data on the substrate properties of the ATP analogues presented here can be rationalized by known binding modes of ATP elucidated by crystal structure analysis (Fig. 5). Whereas, the relevant crystal structures of FAK (with ATP) and Eg5 (with ADP) are available, ${ }^{46}$ no crystal structure of UBA1 in complex with a nucleotide has been reported. Therefore, we used the crystal structure of UBA5, a related E1 for the ubiquitin-like protein Ufm1, in complex with ATP for our comparison. ${ }^{47}$

At first glance, one can see that all positions, at which modification is tolerated by the respective enzymes, point towards the exterior of the protein. In this way, a modification at these positions can be accommodated by the enzyme while the ATP analogue is bound to the active site. Additionally, most of the positions, at which modification is not accepted ( $N 6$ and $C 8$ for FAK and Eg5, $C 2$ and $C 4^{\prime}$ for UBA1), are buried inside the active site excluding modification. The results are thus overall in good agreement with the known crystal structures.

Nevertheless, not all the information deduced from the biochemical experiments can be predicted by the crystal structures. In the case of FAK a large solvent accessible surface can be seen that includes positions $C 2, O 2^{\prime}, O 3^{\prime}$ and $C 4^{\prime}$ of ATP. Whereas all other positions can be modified without loss of function, modification at the $C 4^{\prime}$-position is not tolerated by FAK. Furthermore, identification of the $C 2$-position as the best modification site is not possible solely by inspection of the crystal structure.

Although, a similar solvent accessible surface can be seen in the active site of Eg5 modification at the $\mathrm{O}^{\prime}$-position is more favoured than at the C2-position in this case. Additionally, modification at $C 4^{\prime}$ is tolerated by Eg5. By visual inspection this position seems to be less accessible than in the case of FAK, which does not tolerate modifications at this position.

A very different mode of binding to ATP can be seen in the case of UBA5. In this case, the N6- and $\mathrm{O}^{\prime}$-position are best accessible. Analogues modified at both sites are active in our 
studies using UBA1. Nevertheless, the strong preference for the N6-position cannot be directly rationalized from the crystal structure. Furthermore, modification at position $C 8$ - or position $O 3^{\prime}$ seems to clash similarly with the active site. Whereas the modification at the $O 3^{\prime}$-position is indeed not tolerated, the $C 8$ position can be modified retaining at least some degree of acceptance by UBA1.

Taken together, the biochemical results obtained here add substantially to the knowledge on the active site constraints of the studied enzymes. Since nature has evolved significantly different active site architectures of ATPases ${ }^{3}$ this profiling is of importance to understand substrate recognition and processing. As crystal structures comprise snap-shots of the enzymes bound to ATP, no information can be obtained on perturbations of enzyme-substrate conformations caused by the modification occurring during binding or release. Furthermore, as the ATP binding site of proteins might have a certain degree of flexibility, some modifications clashing in the static crystal structure may be tolerated by active site remodelling. Therefore, the presented biochemical data on acceptance of different ATP analogues in addition to crystal structures will allow an improved design of tailored ATP probes.

\section{Abbreviations}

ATP

FAK

SEM

TMB
Adenosine triphosphate
Focal adhesion kinase
Standard error of the mean
Tetramethylbenzidine

\section{Acknowledgements}

We gratefully acknowledge funding by the Deutsche Forschungsgemeinschaft within the SFB 969 "Chemical and Biological Principals of Cellular Proteostasis". We also acknowledge the Studienstiftung des deutschen Volkes for a stipend to S. M. H. and the Konstanz Research School Chemical Biology for financial support. A. B. is recipient of a fellowship according to the LGFG, Baden-Württemberg, and acknowledges support by the Zukunftskolleg of the University of Konstanz. We acknowledge Naowras Al-Obaidi for purification of full length Eg5. We thank Tobias Strittmatter for helpful discussions.

\section{Notes and references}

1 K. Kikuchi, Chem. Soc. Rev., 2010, 39, 2048.

2 G. A. Ubom, J. B. Hunt and R. B. Timmons, Biochim. Biophys. Acta, Protein Struct. Mol. Enzymol., 1989, 997, 1.

3 (a) I. R. Vetter and A. Wittinghofer, Q. Rev. Biophys., 1999, 32, 1; (b) P. Chene, Nat. Rev. Drug Discovery, 2002, 1, 665.

4 T. Hiratsuka, Biochim. Biophys. Acta, Protein Struct. Mol. Enzymol., 1983, 742, 496.

5 (a) J. C. Cochran, C. A. Sontag, Z. Maliga, T. M. Kapoor, J. J. Correia and S. P. Gilbert, J. Biol. Chem., 2004, 279, 38861; (b) Y.-Z. Ma and E. W. Taylor, J. Biol. Chem., 1997,
272, 724; (c) C. P. Toseland, B. Powell and M. R. Webb, PLoS One, 2012, 7, e38270; (d) D. Q. Ni, J. Shaffer and J. A. Adams, Protein Sci., 2000, 9, 1818; (e) D. Ungureanu, J. Wu, T. Pekkala, Y. Niranjan, C. Young, O. N. Jensen, C.-F. Xu, T. A. Neubert, R. C. Skoda, S. R. Hubbard and O. Silvennoinen, Nat. Struct. Mol. Biol., 2011, 18, 971; (f) I. H. Ganesh, S. Garrod, V. L. Woods Jr and S. S. Taylor, J. Mol. Biol., 2005, 351, 1110.

6 (a) D. I. Garcia, P. Lanigan, M. Webb, T. G. West, J. RequejoIsidro, E. Auksorius, C. Dunsby, M. Neil, P. French and M. A. Ferenczi, Biophys. J., 2007, 93, 2091; (b) A. J. Sowerby, C. K. Seehra, M. Lee and C. R. Bagshaw, J. Mol. Biol., 1993, 234, 114; (c) T. Hiratsuka, Biophys. J., 1997, 72, 843.

7 W. H. Sawjer, R. Y. S. Chan, J. F. Eccleston, B. E. Davidson, S. A. Samat and Y. Yan, Biochemistry, 2000, 39, 5653.

8 (a) F. A. Gonzales, D.-J. Wang, N.-N. Huang and L. A. Heppel, Proc. Natl. Acad. Sci. U. S. A., 1990, 87, 9717; (b) N. G. Kambouris and G. G. Hammes, Proc. Natl. Acad. Sci. U. S. A., 1985, 82, 1950; (c) S. J. Jeng and R. J. Guillory, J. Supramol. Struct., 1975, 3, 448.

9 (a) M. S. Crowder and R. Cooke, Biophys. J., 1987, 51, 323; (b) P. Vogel-Claude, G. Schäfer and W. E. Trommer, FEBS Lett., 1988, 227, 107.

10 J. C. Wolters, G. Roelfes and B. Poolman, Bioconjugate Chem., 2011, 22, 1345.

11 J.-Q. Cheng, W. Jiang and D. D. Hackney, Biochemistry, 1998, $37,5288$.

12 K. Oiwa, J. F. Eccleston, M. Anson, M. Kikumoto, C. T. Davis, G. P. Reid, M. A. Ferenczi, J. E. T. Corrie, A. Yamada, H. Nakayama and D. R. Trentham, Biophys. J., 2000, 78, 3048. 13 K. Oiwa, D. M. Jameson, J. C. Croney, C. T. Davis, J. F. Eccleston and M. Anson, Biophys. J., 2003, 84, 634.

14 (a) Y. Jiang, N. R. Douglas, N. R. Conley, E. J. Miller, J. Frydman and W. E. Moerner, Proc. Natl. Acad. Sci. U. S. A., 2011, 108, 16962; (b) T. Hiratsuka, I. Sakata and K. Uchida, J. Biochem., 1973, 74, 649; (c) H. Strobel, D. Ladant and J.-L. Jestin, J. Mol. Biol., 2003, 332, 1; (d) S. V. Slepenkov and S. N. Witt, FEBS Lett., 2003, 539, 100.

15 D. D. Schlaepfer, C. R. Hauck and D. Sieg, Prog. Biophys. Mol. Biol., 1999, 71, 435.

16 M. T. Valentine and S. P. Gilbert, Curr. Opin. Cell Biol., 2007, 19, 75.

17 I. Lee and H. Schindelin, Cell, 2008, 25(134), 268.

18 A. M. Jawalekar, N. Meeuwenoord, J. G. O. Cremers, H. S. Overkleeft, G. A. van der Marel, F. P. J. T. Rutjes and F. L. van Delft, J. Org. Chem., 2008, 73, 287.

19 H. Staudinger and J. Meyer, Helv. Chim. Acta, 1919, 2, 635. 20 (a) M. Yoshikawa, T. Kato and T. Takenishi, Bull. Chem. Soc. Jpn., 1969, 42, 3505; (b) T. Kovács and L. Ötvös, Tetrahedron Lett., 1988, 29, 4525.

21 A. F. Cook and J. G. Moffat, J. Am. Chem. Soc., 1967, 89, 2697. 22 C. Hörndler, R. J. Suhadolnik, N. F. Muto, E. E. Henderson, M.-X. Guan and W. Pfleiderer, Helv. Chim. Acta, 1997, 80, 767.

23 (a) G. Rangam, N. Z. Rudinger, H. M. Müller and A. Marx, Synthesis, 2005, 1467; (b) T. Strittmatter, J. Aschenbrenner, N. Hardt and A. Marx, ARKIVOC, 2013, 2, 46. 
24 C. R. Noe, J. Winkler, E. Urban, M. Gilbert, G. Haberhauer and H. Brunar, Nucleosides, Nucleotides Nucleic Acids, 2005, $24,1167$.

$25 \mathrm{H}$. Vorbrüggen and C. Ruh-Pohlenz, in Handbook of nucleoside synthesis, Wiley-Interscience, New York, 1st edn, 2001.

26 N. Piton, Y. Mu, G. Stock, T. F. Prisner, O. Schiemann and J. W. Engels, Nucleic Acids Res., 2007, 35, 3128.

27 H. Zhao, A. R. Pagano, W. Wang, A. Shallop, B. L. Gaffney and R. A. Jones, J. Org. Chem., 1997, 62, 7832.

28 A. Gregg, S. E. Bottle, S. M. Devine, H. Figler, J. Linden, P. White, C. W. Pouton, V. Urmaliya and P. J. Scammells, Bioorg. Med. Chem. Lett., 2007, 17, 5437.

29 N. Kohyama, T. Katashima and Y. Yamamoto, Synthesis, 2004, 2799.

30 G. Manning, D. B. Whyte, R. Martinez, T. Hunter and S. Sudarsanam, Science, 2002, 298, 1912.

$31 \mathrm{~J}$. Baselga, Science, 2006, 312, 1175.

32 S. K. Mitra and D. D. Schlaepfer, Curr. Opin. Cell Biol., 2006, $18,516$.

33 A. Buntru, K. Kopp, M. Voges, R. Frank, V. Bachmann and C. R. Hauck, J. Biol. Chem., 2011, 286, 9555.

34 K. E. Sawin, K. LeGuellec, M. Philippe and T. J. Mitchison, Nature, 1992, 359, 540.

35 C. L. Asbury, Curr. Opin. Cell Biol., 2005, 17, 89.

36 T. U. Mayer, T. M. Kaporr, S. J. Haggarty, R. W. King, S. L. Schreiber and T. J. Mitchison, Science, 1999, 286, 971.

37 (a) T. P. Geladopoulos, T. G. Sotiroudis and A. E. Evangelopoulos, Anal. Biochem., 1991, 192, 112; (b) M. Catarinella, T. Grüner, T. Strittmatter, A. Marx and T. U. Mayer, Angew. Chem., Int. Ed., 2009, 48, 9072.

38 (a) R. J. Deshaies and C. A. Joazeiro, Annu. Rev. Biochem., 2009, 78, 399; (b) T. Ravid and M. Hochstrasser, Nat. Rev. Mol. Cell Biol., 2008, 9, 679; (c) D. Rotin and S. Kumar, Nat. Rev. Mol. Cell Biol., 2009, 10, 398; (d) R. L. Welchman, C. Gordon and R. J. Mayer, Nat. Rev. Mol. Cell Biol., 2005, 6, 599.

39 (a) Y. Yang, J. Kitagaki, R.-M. Dai, Y. C. Tsai, K. L. Lorick, R. L. Ludwig, S. A. Pierre, J. P. Jensen, I. V. Davydov, P. Oberoi, C.-C. H. Li, J. H. Kenten, J. A. Beutler, K. H.Vousden and A. M. Weissman, Cancer Res., 2007, 67, 9472; (b) G. W. Xu, M. Ali, T. E. Wood, D. Wong,
N. Maclean, X. Wang, M. Gronda, M. Skrtic, X. Li, R. Hurren, X. Mao, M. Venkatesan, R. Zavareh, T. Ketela, J. C. Reed, D. Rose, J. Moffat, R. A. Batey, S. Dhe-Paganon and A. D. Schimmer, Blood, 2010, 115, 2251.

40 (a) A. Ciechanover, Y. Hod and A. Hershko, Biochem. Biophys. Res. Commun., 1978, 81, 1100; (b) A. Hershko and A. Ciechanover, Annu. Rev. Biochem., 1998, 67, 425; (c) M. Scheffner, U. Nuber and J. M. Huibregtse, Nature, 1995, $373,81$.

41 U. Nuber, S. E. Schwarz and M. Scheffner, Eur. J. Biochem., 1998, 254, 643.

42 (a) M. Scheffner and O. Staub, BMC Biochem., 2007, 8(Suppl. 1), 6; (b) A. Hogart, D. Wu, J. M. LaSalle and N. C. Schanen, Neurobiol. Dis., 2010, 38, 181; (c) S. E. Smith, Y. D. Zhou, G. Zhang, Z. Jin, D. C. Stoppel and M. P. Anderson, Sci. Transl. Med., 2011, 3, 103ra97.

43 L. M. Elphick, S. E. Lee, V. Gouverneur and D. J. Mann, ACS Chem. Biol., 2007, 2, 299.

44 (a) K. Shah, Y. Liu, C. Deirmengian and K. M. Shokat, Proc. Natl. Acad. Sci. U. S. A., 1997, 94, 3565; (b) T. M. Kapoor and T. J. Mitchison, Proc. Natl.Acad. Sci. U. S. A., 1999, 96, 9106.

45 (a) T. A. Soucy, P. G. Smith, M. A. Milhollen, A. J. Berger, J. M. Gavin, S. Adhikari, J. E. Brownell, K. E. Burke, D. P. Cardin, S. Critchley, C. A. Cullis, A. Doucette, J. J. Garnsey, J. L. Gaulin, R. E. Gershman, A. R. Lublinsky, A. McDonald, H. Mizutani, U. Narayanan, E. J. Olhava, S. Peluso, M. Rezaei, M. D. Sintchak, T. Talreja, M. P. Thomas, T. Traore, S. Vyskocil, G. S. Weatherhead, J. Yu, J. Zhang, L. R. Dick, C. F. Claiborne, M. Rolfe, J. B. Bolen and S. P. Langston, Nature, 2009, 458, 732; (b) J. J. Chen, C. A. Tsu, J. M. Gavin, M. A. Milhollen, F. J. Bruzzese, W. D. Mallender, M. D. Sintchak, N. J. Bump, X. Yang, J. Ma, H.-K. Loke, Q. Xu, P. Li, N. F. Bence, J. E. Brownell and L. R. Dick, J. Biol. Chem., 2011, 286, 40867.

46 (a) D. Lietha, X. Cai, D. F. Ceccarelli, Y. Li, M. D. Schaller and M. J. Eck, Cell, 2007, 129, 1177; (b) J. Turner, R. Anderson, J. Guo, C. Beraud, R. Fletterick and R. Sakowicz, J. Biol. Chem., 2001, 276, 25496.

47 J. P. Bacik, J. R. Walker, M. Ali, A. D. Schimmer and S. DhePaganon, J. Biol. Chem., 2010, 285, 20273. 